\title{
A phase III, open label, randomized multicenter controlled trial of oral versus intravenous treosulfan in heavily pretreated recurrent ovarian cancer: a study of the North-Eastern German Society of Gynecological Oncology (NOGGO)
}

\author{
Jalid Sehouli $^{1}$ - Oliver Tomè ${ }^{2}$ - Desislava Dimitrova ${ }^{1} \cdot$ Oumar Camara $^{3} \cdot$ Ingo Bernhard Runnebaum $^{4}$. \\ Hans Werner Tessen ${ }^{5} \cdot$ Beate Rautenberg $^{6} \cdot$ Radoslav Chekerov $^{1} \cdot$ Mustafa Zelal Muallem $^{1} \cdot$ Michael Patrick Lux $^{7}$. \\ Tanja Trarbach ${ }^{8} \cdot$ Gerald Gitsch $^{6}$
}

Received: 27 June 2016 / Accepted: 14 November 2016 / Published online: 28 November 2016

(c) The Author(s) 2016. This article is published with open access at Springerlink.com

\begin{abstract}
Objective In recurrent ovarian cancer (ROC), there is a high demand on effective therapies with a mild toxicity profile. Treosulfan is an alkylating agent approved as oral (p.o.) and intravenous (i.v.) formulation for the treatment of recurrent ovarian cancer. Data on safety and efficacy for either formulation are rare. For the first time we conducted a randomized phase III study comparing both formulations in women with ROC.

Methods Patients having received at least two previous lines of chemotherapy were randomly assigned to one of two treatment arms: treosulfan i.v. $7000 \mathrm{mg} / \mathrm{m}^{2} \mathrm{~d} 1 \mathrm{q} 4 \mathrm{w}$ or treosulfan p.o. $600 \mathrm{mg} / \mathrm{m}^{2}$ d1-28 q8w. Primary endpoint
\end{abstract}

Results of this study were presented at the IGCS 2014 in Melbourne.

iOMEDICO Clinical Research, Freiburg, Germany is associated to University Hospital Essen, West German Cancer Center, Clinic for Internal Medicine (Tumor Research), Essen, Germany.

Electronic supplementary material The online version of this article (doi:10.1007/s00432-016-2307-0) contains supplementary material, which is available to authorized users.

Jalid Sehouli

jalid.sehouli@charite.de

1 Gynecology and Gynecologic Oncology, Charité University Medicine Campus Virchow, Augustenburger Platz 1, 13353 Berlin, Germany

2 Gynecologic Cancer Centre Karlsruhe, St. Vincentius Clinics, Karlsruhe, Germany

3 Centre for Gynecology, Hufeland Clinic, Bad Langensalza, Germany

4 Clinic for Gynecology and Obstetrics, University Clinic, Jena, Germany was safety regarding hematological and gastrointestinal toxicity grade III/IV, secondary endpoints were other toxicities, clinical benefit rate (CBR), time to progression (TTP), overall survival (OS) and quality of life.

Results 250 patients were treated with treosulfan i.v. (128) or treosulfan p.o. (122). In general treosulfan therapy was well tolerated in both treatment arms. Leukopenia grade III/IV occurred significantly more frequently in the p.o. arm (3.9\% i.v. arm, $14.8 \%$ p.o. arm, $p=0.002)$. Other toxicities were similar in both arms. CBR was comparable between arms (41.4\% i.v. arm, $36.9 \%$ p.o. arm). No difference in TTP (3.7 months i.v. arm, 3.5 months p.o. arm) or OS (13.6 months i.v. arm, 10.4 months p.o. arm, $p=0.087$ ) occurred.

Conclusions Given the safety and efficacy results treosulfan is an acceptable option for heavily pretreated OC patients. Regarding the toxicity profile the i.v. application was better tolerated with less grade III and IV toxicities.

Keywords Treosulfan · Oral versus intravenous · Ovarian cancer $\cdot$ Recurrent

Oncology Practise, Goslar, Germany

6 Gynecology and Gynecologic Oncology, University Clinic, Freiburg, Germany

7 Department of OOGYN, Gyncecological University Cancer Center of Franconia, University Hospital Erlangen, CCC Erlangen-EMN, Erlangen, Germany

8 iOMEDICO Clinical Research, Freiburg, Germany 


\section{Introduction}

In Europe, ovarian cancer is the fifth most common cancer and one of the five leading malignancies responsible for cancer-related deaths among females (Ferlay et al. 2013). As the results of EUROCARE-5 study demonstrated the European mean age-standardized 5-year survival for ovarian cancer is low-37.6\% (De Angelis et al. 2014). About 66,000 women in Europe were diagnosed with ovarian cancer and estimated 42,000 died of this disease in 2012 (Ferlay et al. 2013). Primary treatment of ovarian cancer includes cytoreductive surgery followed by systemic chemotherapy. Standard first-line chemotherapy is based on platinum and taxanes-eventually in combination with bevacizumab-which achieves high response rates up to $80 \%$ (McGuire et al. 1996; Pujade-Lauraine et al. 2010). However, in the majority of patients the disease recurs and further therapy is required. Drug-resistance to platinum-based chemotherapy is one of the most difficult clinical situations because the effect of the current therapies is very limited and the outcome is poor. There is a need for implementation of new chemotherapeutic agents for further treatment. Treosulfan $\left(\right.$ Ovastat $\left.^{\circledR}\right)$ is a bifunctional prodrug of an alkylating cytotoxic agent that is licensed in several European countries for the treatment of ROC (Gropp et al. 1998; Hilger et al. 2000). Treosulfan also shows activity in other solid tumors and hematologic malignancies (Boztug et al. 2014; Köpf-Maier and Sass 1996). It is known for its modest toxicity profile as demonstrated in previous phase II and III studies (Reed et al. 2006; Kledsen et al. 1998). With a relative bioavailability of $97 \%$, treosulfan p.o. has shown a nearly equivalent bioavailability $\left(\mathrm{AUC}_{\text {oral }}=82.1 \pm 39.4 \mu \mathrm{g} / \mathrm{ml} \mathrm{h}\right)$ compared to treosulfan i.v. $\left(\mathrm{AUC}_{\mathrm{i} . \mathrm{v}}=85.4 \pm 30.3 \mu \mathrm{g} / \mathrm{ml} \mathrm{h}\right)$ in ovarian cancer patients (Hilger et al. 2000). Till now data on safety and efficacy for either formulation in heavily pretreated patients with ovarian cancer are rare. Therefore, for the very first time, we conducted a randomized phase IIIb study comparing the intravenous versus oral formulation of treosulfan in women with recurrent ovarian cancer.

\section{Patients and methods}

This open label, randomized, controlled, multicenter phase IIIb trial was conducted at 30 institutions in Germany. Patients were eligible for enrollment, if they had histologically confirmed ROC and had received at least two previous lines of chemotherapy. After inclusion of 18 patients an amendment inured, stating that induction and re-induction of platinum-containing pretreatments were counted as one treatment line. A further amendment was implemented after the inclusion of 85 patients stating that also patients with more than two previous treatment lines could be included, and induction and re-induction therapy were counted as separate treatment lines. For data analysis, induction and re-induction therapy lines were counted as one therapy line. Written informed consent was given by all patients before enrollment. For patient inclusion following criteria needed to be met: (1) two-dimensional measurable tumor lesion or progressive disease evaluable by increased CA125 $>100$ $\mathrm{U} / \mathrm{ml}$ ); (2) life expectancy of at least 3 months; (3) Karnofsky index $>50 \%$; (4) adequate organ functions, defined as leukocyte count $\geq 3.5$ cells $\times 10^{9} / 1$, platelet count $\geq 100 \times 10^{9} / 1$, creatinine and total bilirubin $1.25 \times$ the upper limit of the normal range or less; (5) no prior treatment with treosulfan; (6) no second malignancy (except basilioma or cervical cancer in situ); (7) no ascites/pleural effusion without evaluable or measurable tumor lesion or not elevated CA125; (8) no concurrent radiotherapy or antineoplastic therapy.

Patients were randomly assigned to one of the two treatment arms: treosulfan i.v. $7000 \mathrm{mg} / \mathrm{m}^{2} \mathrm{~d} 1 \mathrm{q} 4 \mathrm{w}$ or treosulfan p.o. $600 \mathrm{mg} / \mathrm{m}^{2} \mathrm{~d} 1-28 \mathrm{q} 8 \mathrm{w}$. Treatment was continued until tumor progression, unacceptable toxicity or treatment delay for more than 2 weeks. Patients with a complete remission received optional chemotherapy for two more months before therapy was terminated. Supportive care was allowed and given individually according to clinical requirements. Patients in both arms needed to have a leukocyte count of at least 3500 cells $/ \mu$ and a platelet count of at least $100,000 /$ $\mu \mathrm{l}$ to receive subsequent cycles of chemotherapy. Treosulfan dose had to be reduced for $1000 \mathrm{mg} / \mathrm{m}^{2}$ body surface for i. v. administration or one capsule for oral administration in the next treatment cycle, if leukocytes dropped below 1000 cells $/ \mu$ or platelets dropped below $25,000 / \mu \mathrm{l}$ upon administration of treosulfan. Re-escalation was not allowed. For patients with renal insufficiency receiving treosulfan i.v., treatment-dose was reduced to $6000 \mathrm{mg} / \mathrm{m}^{2}$, if creatinine clearance was $20-40 \mathrm{ml} / \mathrm{min}$ and to $5000 \mathrm{mg} /$ $\mathrm{m}^{2}$ if creatinine clearance was less than $20 \mathrm{ml} / \mathrm{min}$. The primary endpoint of the study was the comparison of safety of both schemes regarding hematological and gastrointestinal toxicity grade III and IV. Adverse events were assessed continuously by patient questioning, physical examination and evaluation of laboratory results, and graded according to the National Cancer Institute Common Toxicity criteria (CTC) version 2.0. Secondary endpoints were comparison of other adverse events, clinical benefit rate (CBR), time to progression (TTP), overall survival (OS) and quality of life. Response was evaluated by assessment of two-dimensional measurable lesions and determination of CA125 levels. Lesions were examined every 3 months using imaging procedures. CA125 levels were determined every month. A complete response was defined as complete disappearance 
of tumor lesions. A partial response was defined as $50 \%$ or more decrease in tumor size. Assessments had to be confirmed after at least 4 weeks. No change/stable disease was defined as less than $50 \%$ decrease or less than $25 \%$ increase in tumor size or of reference metastasis. Progressive disease was defined as appearance of any new lesions or growth of more than $25 \%$ or increase in CA125 level by more than $25 \%$. This increase had to be confirmed after at least 2 weeks. TTP was defined as the time from the date of randomization to the date of first documented progression. Patients not experiencing PD were censored with the last date of either tumor evaluation, measurement of CA125 or with the start of a new therapy. Survival was defined as the time from date of therapy start to date of death. Patients who were alive at the end of the study were censored at the last date where they were known to be alive. Platinumsensitivity was defined by a relapse-free period of more than 6 months following platinum-based chemotherapy. Platinum-sensitivity was calculated using the relapse-free interval from end of the last platinum-containing therapy until start of the following therapy (Hanker et al. 2012). For the analyses of treatment duration, every cycle started was calculated as a full cycle. Quality of life was assessed using the EORTC QLQ-C30 questionnaire. Patients were asked to complete the questionnaire before first cycle of study therapy and consecutively during treatment every other month. The last questionnaire was completed at the end of treatment visit. Once patients discontinued therapy because of complete remission or intolerable toxicity, disease status was assessed every 3 months. After disease progression follow-up was only performed on survival data.

\section{Statistical analysis}

The sample size was planned to detect a $15 \%$ difference in the incidence of grade 3/4 adverse events of hematological or gastrointestinal origin between the two treatment arms. An event was assumed to occur with a probability of $25 \%$. Calculation was based on a two-sided test with $\alpha=0.05$ and $80 \%$ power. As no clear hypothesis was stated, all $p$ values are explorative. There was no adjustment for multiple testing. A total number of 270 patients (129 per treatment arm plus $4 \%$ dropouts) were planned to be recruited. All analyses were based on the safety population including all patients who received at least one dose of study medication. Differences in proportions of adverse events and response were analyzed using a twosided Fisher's exact test or two-sided $\chi^{2}$ test. Clinical benefit rate (CBR) was defined as sum of complete remission $(\mathrm{CR})$, partial remission (PR) and no change (NC) as best response $(\mathrm{CBR}=\mathrm{CR}+\mathrm{PR}+\mathrm{NC})$. Time to progression (TTP) and overall survival time (OS) were estimated by use of Kaplan-Meier, and differences were compared using the log-rank test. Subgroup analyses were performed retrospectively. Hazard ratios were calculated using cox and logistic models. Effects of possible confounders were estimated using multivariate cox or logistic regression modeling. For the calculation of quality of life scores, scoring manuals were applied. All analyses were performed using STATISTICA version 10 or R 2.15.1.

\section{Results}

\section{Patient characteristics}

Between November 2002 and January 2014, 265 patients were enrolled at 30 study sites. Patient consort diagram is shown in Fig. 1. 250 patients (128 treosulfan i.v. and 122 treosulfan p.o.) received at least one dose of treosulfan and were evaluable (safety set). Patient characteristics are shown in Table 1. There were no substantial differences between both treatment arms. Patients received two previous chemotherapies in median. As first-line therapy $82 \%$ of patients received a platinum-containing regimen combined with paclitaxel. As second-line therapy topotecan or a platinum-containing regimen were administered in about $45 \%$ of patients. The median number of concomitant diseases was three. Most important concomitant diseases are listed in Table 1.

\section{Treatment}

The mean number of administered treatment cycles was 4.4 (95\% CI 3.7-5.1; median 3.0) in the i.v. arm and 2.3 (95\% CI 1.9-2.8; median 2.0) in the p.o. arm. Considering that i.v. and p.o. treatment cycles are of different length ( 28 days for the i.v. arm and 56 days for the p.o. arm) treatment duration was comparable in both treatment arms. $64 \%$ (160 of 250) of patients continued study treatment until disease progression as scheduled in the protocol. Unforeseen treatment discontinuations were limited and comparable in both treatment arms $(27.3 \%$ in the i.v. arm and $30.3 \%$ in the p.o. arm). The proportion of discontinuations due to toxicity was significantly higher in the p.o. treatment arm $(17.2 \%)$ than in the i.v. treatment $\operatorname{arm}(6.3 \% ; p=0.0094)$ (Fig. 1). The median dose intensity in the i.v. arm was $97.8 \%$ (25-75\% quartile, $90.0-100.8 \%$ ) and $99.6 \%$ on the p.o. arm (25-75\% quartile, $84.0-124.4 \%)$.

\section{Adverse events/toxicity}

Adverse events occurred in $91.2 \%$ of patients $(90.6 \%$ in the i.v. arm and $91.8 \%$ in the p.o. arm). Toxicities of grade III/ IV were rare. 25.0 and $30.3 \%$ of patients in the i.v. and p.o. 
Fig. 1 CONSORT diagram. i.v., intravenous; p.o., per os/ oral

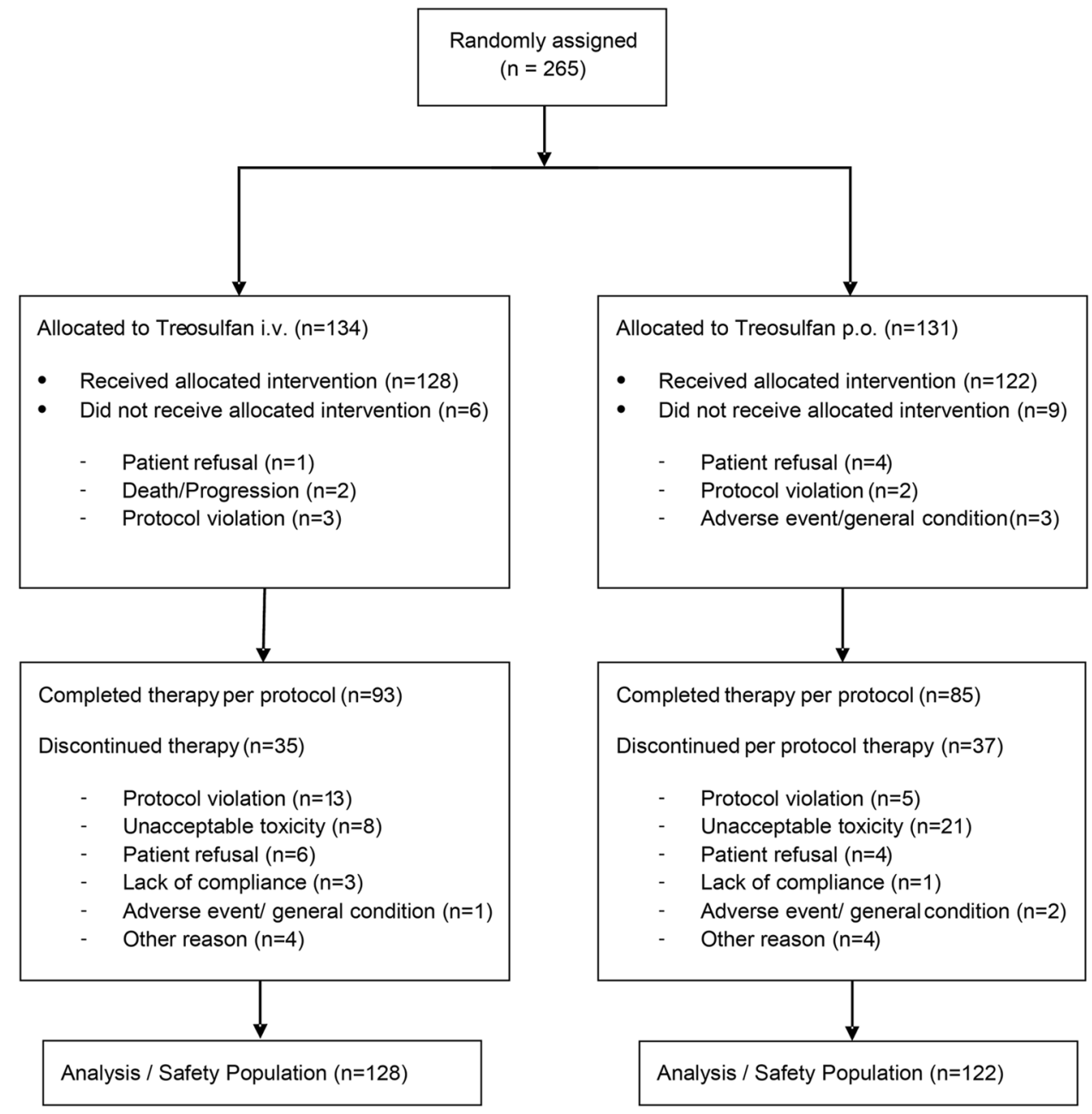

arm experienced serious adverse events, respectively. Frequently reported hematological and non-hematological toxicities of all grades are listed in Table 2. Hematological and gastrointestinal toxicities were the main grade III/IV toxicities and were observed in $17.6 \%$ (12.5\% in the i.v. arm and $22.1 \%$ in the p.o. arm) and $18.0 \%(17.9 \%$ in the i.v. arm and $18.0 \%$ in the p.o. arm) of patients, respectively. The severity of hematological events differed between treatment arms. Although the total number of reported hematological events of all grades was similar in both arms (40.6\% in the i.v treatment arm, $39.3 \%$ in the p.o. treatment arm), grade III/IV events occurred more often in the p.o. arm $(22.1 \%$ vs. $12.5 \%$ in the i.v. arm). The frequency of grade III/IV leukopenia was significantly higher in the p.o. treatment arm than in the i.v. arm (14.8\% in the p.o. arm and $3.9 \%$ in the i.v. arm, $p=0.0038$ ). However, only one patient in the p.o. arm experienced a febrile neutropenia grade III. In addition to hematological and gastrointestinal toxicities, constitutional symptoms and pain were recorded most frequently. Alopecia occurred in $19(7.6 \%)$ of patients. Almost all cases were grade I events, only one patient suffered from alopecia grade II. No grade III alopecia occurred. Except the above mentioned, no differences in hematological and non-hematological toxicities of any grade between both arms were observed. 17 patients (11 in the i.v. arm and 6 in the p.o. arm) died during study period. In all cases, death was concerned as not related to study medication. Underlying malignant disease was stated as cause of death for ten patients in the i.v. arm and five patients in the p.o. arm. Two patients, one in the i.v arm and one in the p.o. arm died of thromboembolic events (pulmonary embolism in the p.o. arm and stroke in the i.v. arm) not considered to be caused by the study medication.

\section{Efficacy}

Response was evaluable in 212 patients (111 in the i.v. arm and 101 in the p.o. arm). Non-evaluable patients mainly resulted from early treatment discontinuations, when no response assessment was performed. CBR was $39.2 \%$ (95\% CI 33.2-45.6) and was comparable between both arms (treosulfan i.v.: 41.4, 95\% CI 32.9-50.5; treosulfan 
Table 1 Baseline

characteristics

Treosulfan i.v. $n=128$ Treosulfan p.o. $n=122$

\begin{tabular}{|c|c|c|}
\hline Age in years at therapy start [median (range)] & $63(32-84)$ & $64(29-87)$ \\
\hline \multicolumn{3}{|l|}{ Karnofsky index } \\
\hline $100 \%$ & $37(28.9)$ & $36(29.5)$ \\
\hline $90 \%$ & $33(25.8)$ & $27(22.1)$ \\
\hline $80 \%$ & $33(25.8)$ & $36(29.5)$ \\
\hline$\leq 70 \%$ & $12(9.4)$ & $11(9.0)$ \\
\hline Not specified/missing & $13(10.2)$ & $12(9.8)$ \\
\hline \multicolumn{3}{|l|}{ FIGO stage at initial diagnosis } \\
\hline I & $5(3.9)$ & $5(4.1)$ \\
\hline II & $5(3.9)$ & $2(1.6)$ \\
\hline III & $73(57.0)$ & $60(49.2)$ \\
\hline IV & $30(23.4)$ & $29(23.8)$ \\
\hline Not specified & $15(11.7)$ & $26(21.3)$ \\
\hline \multicolumn{3}{|l|}{$\begin{array}{l}\text { Relapse-free interval after last platinum-containing } \\
\text { therapy }\end{array}$} \\
\hline$<6$ months & $68(53.1)$ & $65(53.3)$ \\
\hline$>6$ months & $60(46.9)$ & $57(46.7)$ \\
\hline \multicolumn{3}{|l|}{ Previous therapies } \\
\hline Surgery (initial) & $127(99.2)$ & $122(100.0)$ \\
\hline Radiotherapy & $9(7.0)$ & $7(5.7)$ \\
\hline $\begin{array}{l}\text { Number of previous chemotherapy lines [median } \\
\text { (range)] }\end{array}$ & $2(1-7)$ & $2(1-6)$ \\
\hline 2 previous lines & $81(63.3)$ & $80(65.6)$ \\
\hline$\geq 2$ previous lines & $47(36.7)$ & $42(34.4)$ \\
\hline Concomitant diseases & $3(1-13)$ & $3(1-11)$ \\
\hline Gastrointestinal disorders & $62(48.4)$ & $68(55.3)$ \\
\hline Vascular disorders & $63(49.2)$ & $62(50.4)$ \\
\hline Hypertension & $42(32.8)$ & $49(39.8)$ \\
\hline Thromboembolism & $12(9.4)$ & $7(5.7)$ \\
\hline Metabolism/nutrition and endocrine disorders & $59(46.1)$ & $62(50.4)$ \\
\hline Diabetes & $8(6.3)$ & $11(8.9)$ \\
\hline Respiratory disorders & $28(21.9)$ & $23(18.7)$ \\
\hline Thromboembolism & $5(3.9)$ & $6(4.9)$ \\
\hline Cardiac disorders & $12(9.4)$ & $20(16.3)$ \\
\hline Cardiac failure & $4(3.1)$ & $4(3.3)$ \\
\hline Coronary artery disease & $2(1.6)$ & $3(2.4)$ \\
\hline
\end{tabular}

Data are presented as $n(\%)$ unless specified separately; FIGO, International Federation of Gynecology and Obstetrics; study sites could choose to document either FIGO or TNM stage, TNM documentation was translated into FIGO (according to NCCN guidelines version 3.2014 on epithelial ovarian cancer)

p.o.: $36.9,95 \%$ CI $28.5-46.1 ; p=0.6063$ ) (Table 3). The median follow-up was 8.6 months (10.0 months in the i.v. arm and 7.6 months in the p.o. arm). We could not detect any significant difference in the median TTP and median OS between both treatment arms (Figs. 2a, 3a). However, a nonsignificant trend toward longer survival in the i.v. arm was observed. Median TTP was 3.7 months (95\% CI 2.94.8 ) in the i.v. treatment arm and 3.5 months (95\% CI 2.84.4) in the p.o. treatment arm (Fig. 2a; HR 1.07, 95\% CI $0.81-1.41, p=0.634)$. Median OS was 13.6 months $(95 \%$
CI 11.3-16.8) in the i.v. treatment arm and 10.4 months (95\% CI 8.6-12.8) in the p.o. treatment arm (Fig. 2b; HR $1.28,95 \%$ CI $0.96 .1 .70, p=0.0873$ ).

Subgroup analyses on efficacy parameters were performed using three models, each of them exploring the impact of the following covariates on CBR, TTP and OS: route of administration (p.o. vs. i.v.), platinum-sensitivity (platinum-sensitive vs. platinum-resistant), number of previous therapy lines ( $>2$ lines vs. $\leq 2$ lines) and Karnofsky index (<90 vs. $\geq 90)(\mathrm{S} 1 \mathrm{~A}-\mathrm{C})$. The model revealed 
Table 2 Hematological and non-hematological toxicities all grades, highest grade per patient

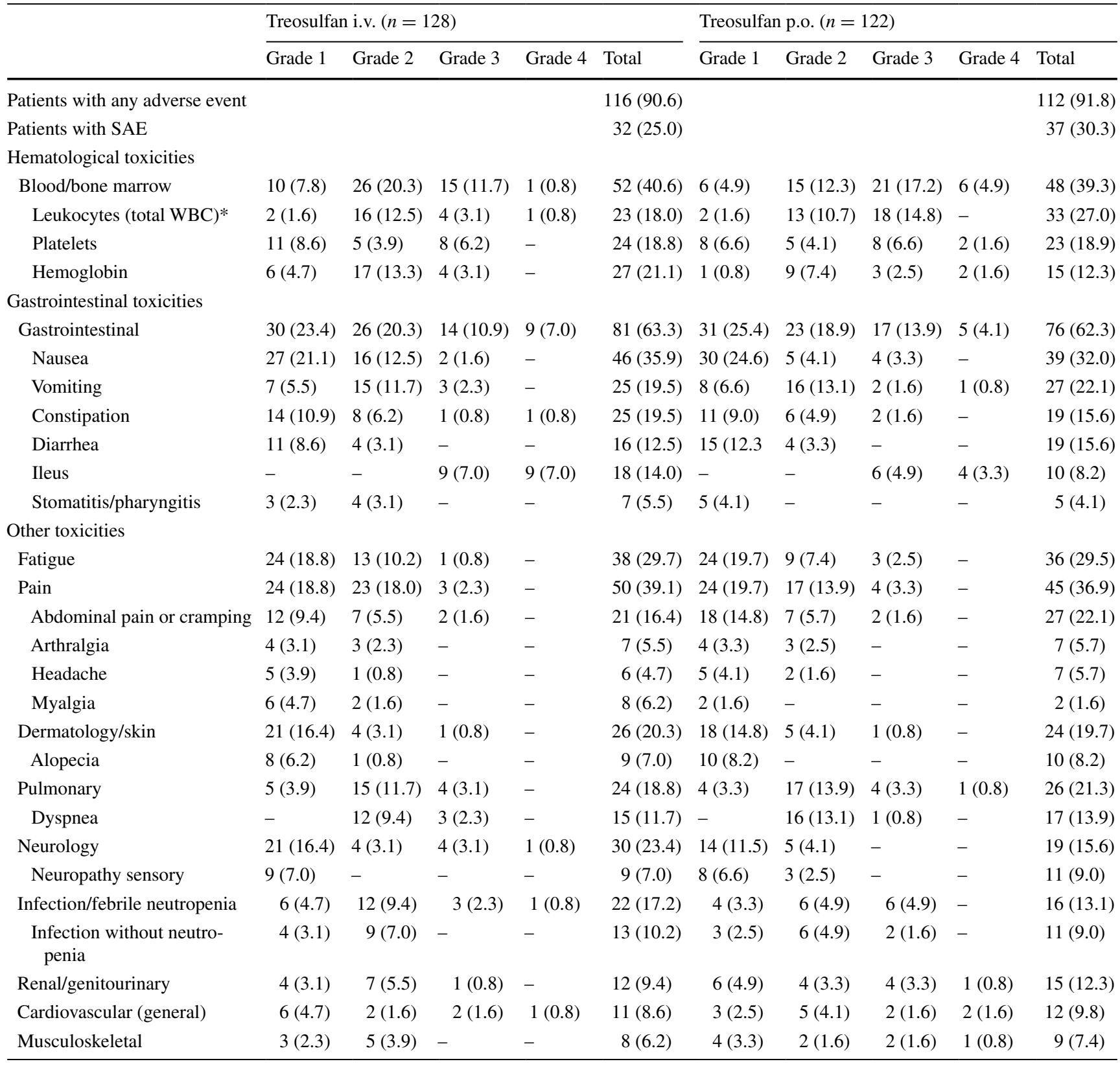

Data are presented as $n(\%)$, adverse events were graded according to the National Cancer Institute Common Toxicity Criteria version 2.0

* Events occurring with a frequency $>5 \%$ in any grade are listed

significant differences for the following subgroups: Platinum-sensitive patients showed a higher CBR (47.9\% vs. 31.6; $p=0.003$ ) (S1 A) and developed a longer TTP (5.0 vs. 2.8 months; $p<0.0001$ ) (Fig. 2b and S1 B) compared to platinum-resistant patients. In the subgroup of Karnofsky index, the model revealed a significant longer TTP and OS for patients with a Karnofsky index $\geq 90$ (TTP: 4.3 vs. 3.0, $p=0.039$ and OS: 15.1 vs. $8.6, p<0.0001)$ (S1 B, C). For platinum-sensitivity, no differences in OS were observed. The model did not show significant differences for any efficacy parameter for the subgroup of number of previous therapy lines and the subgroup of route of administration.

$58 \%$ of patients received at least one further therapy after completion of study therapy. $11.6 \%$ of subsequent therapies were platinum based.

\section{Quality of life/patient reported outcome}

Questionnaire compliance was comparable in both treatment arms. Baseline questionnaires were available for 
Table 3 Response evaluation

\begin{tabular}{lclc}
\hline CBR $(95 \%$ CI $)$ & Treosulfan i.v. $n=128$ & Treosulfan p.o. $n=122$ & Total \\
& $53(41.4(32.9-50.5))$ & $45(36.9(28.5-46.1))$ & $98(39.2(33.2-45.6))$ \\
\hline CR & $5(3.9)$ & $1(0.8)$ & $6(2.4)$ \\
PR & $16(12.5)$ & $15(12.3)$ & $31(12.4)$ \\
NC & $32(25.0)$ & $29(23.8)$ & $61(24.4)$ \\
PD & $58(45.3)$ & $56(45.9)$ & $114(45.6)$ \\
NE & $17(13.3)$ & $21(17.2)$ & $38(15.2)$ \\
\hline
\end{tabular}

Best response per patient. Data are presented as $n(\%)$

$C B R$ Clinical benefit rate; $C R$ Complete response; $P R$ Partial response; $N C$ No change; $P D$ Progressive disease; $N E$ Not evaluable

$81.3 \%$ of patients in the i.v. treatment arm and from $82.0 \%$ of patients in the p.o. treatment arm. Significant differences in global health, fatigue as well as in gastrointestinal and hematological subscales were detected neither between the treatment arms nor over time during the first 6 months of treatment (S2 A-F).

\section{Discussion}

As most ovarian cancer patients suffer from relapse, treatment of recurrent ovarian cancer remains a major issue in the clinical management of ovarian cancer. Standard chemotherapies are either platinum-based regimens in combination with agents such as liposomal doxorubicin, gemcitabin, paclitaxel and bevacizumab or olaparib (Pujade-Lauraine et al. 2010; Aghajanian et al. 2012; Oza et al. 2015) or non-platinum-based regimens such as liposomal doxorubicin, paclitaxel, topotecan and gemcitabine combined with targeted therapies like bevacizumab (Meier et al. 2009; Monk et al. 2010; Pujade-Lauraine et al. 2014; Sehouli et al. 2008). In general, patients with more than two previous lines of chemotherapy have been excluded from these trials, so translation of the results on heavily pretreated patients is limited. Various randomized and non-randomized trials have demonstrated activity in recurrent ovarian cancer for the alkylating agent treosulfan (Gropp et al. 1998; Köpf-Meier and Sass 1996; Meier et al. 2009). For patients in the palliative setting of recurrent ovarian cancer the maintenance of quality of life is, beside the maintenance of tumor control, the main goal of treatment. To sustain a maximum of flexibility, the choice of the route of administration is an important aspect in everyday life. Liu et al. (1997) assessed patient preferences for oral versus intravenous palliative chemotherapy using a structured interviewer-administered questionnaire. Major reasons for a clear preference for oral chemotherapy (92 of 103 patients) were convenience, problems with i.v. access and a better chemotherapy-taking environment outside the clinic. Nevertheless, despite their initial clear preference for oral chemotherapy patients were not willing to sacrifice efficacy for their preference. In contrast, the NOGGO study group has investigated the preference of elderly patients $\geq 65$ years regarding p.o. or i.v. treosulfan for the treatment of relapsed ovarian cancer in 123 patients and showed a clear preference for the i.v. administration of treosulfan. The main reasons for this decision were the hope for less gastrointestinal toxicities, a better control of drug delivery and the assumption, that i.v. therapy is more effective. Therefore, in terms of different patient populations and their preferences the availability of different formulations might be worthwhile, if efficacy and safety are comparable. Here, we present the final results of our randomized study comparing p.o. and i.v. treosulfan to increase information on safety and efficacy of both formulations. Previous data especially on p.o. treosulfan were mainly based on a low number of patients. Detailed safety data were scarce. To our knowledge, our study is the largest performed in ovarian cancer comparing i.v. versus p.o. treosulfan in terms of efficacy and safety.

Despite the heavy pretreatment of the patients, treosulfan therapy was well tolerated in both treatment arms. The majority of toxicities were grade I or II. Most frequently observed hematological toxicities of grade III/IV in both treatment arms were leucopenia $(9.2 \% ; 3.9 \%$ in the i.v. arm and $14.8 \%$ in the p.o. arm) and thrombocytopenia (7.2\%; $6.2 \%$ in the i.v. arm and $8.2 \%$ in the p.o. arm). Compared to the i.v. arm, a statistically significant higher number of grade III/IV leucopenia occurred in the p.o. arm in our study. This number might explain the higher number of treatment discontinuations in this treatment arm due to unacceptable toxicity. However, only one case of febrile neutropenia in the p.o. treatment arm was reported. Most frequent documented gastrointestinal toxicity grade III/IV was ileus $(11.2 \% ; 14.0 \%$ in the i.v. arm and $8.2 \%$ in the p.o. arm). Toxicities during therapy with intravenous treosulfan had been reported in several previous studies (Meier et al. 2009; Mahner et al. 2012). However, safety data on therapy with oral treosulfan are rare. Keldsen et al. (1998) reported that oral treosulfan treatment is well tolerated as 


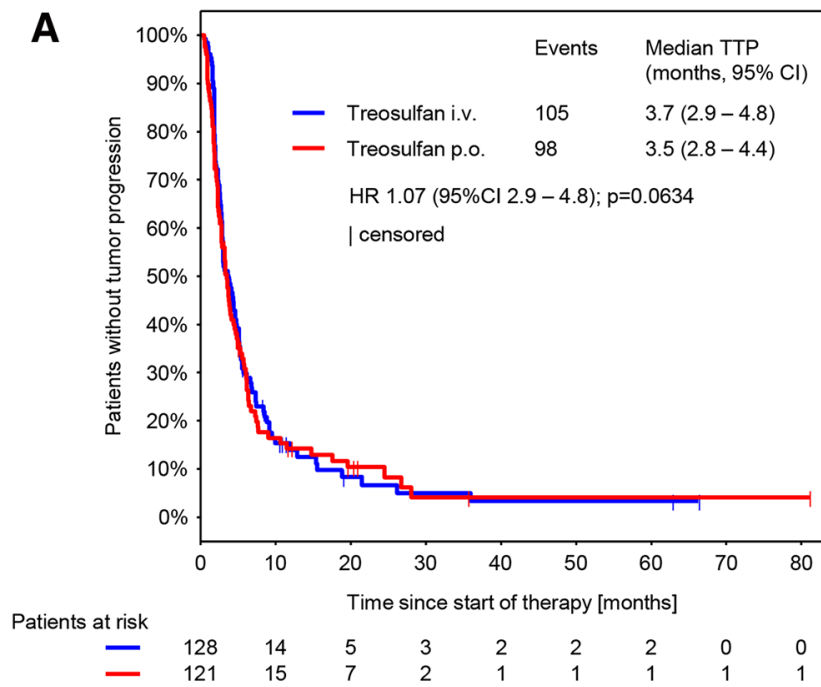

B
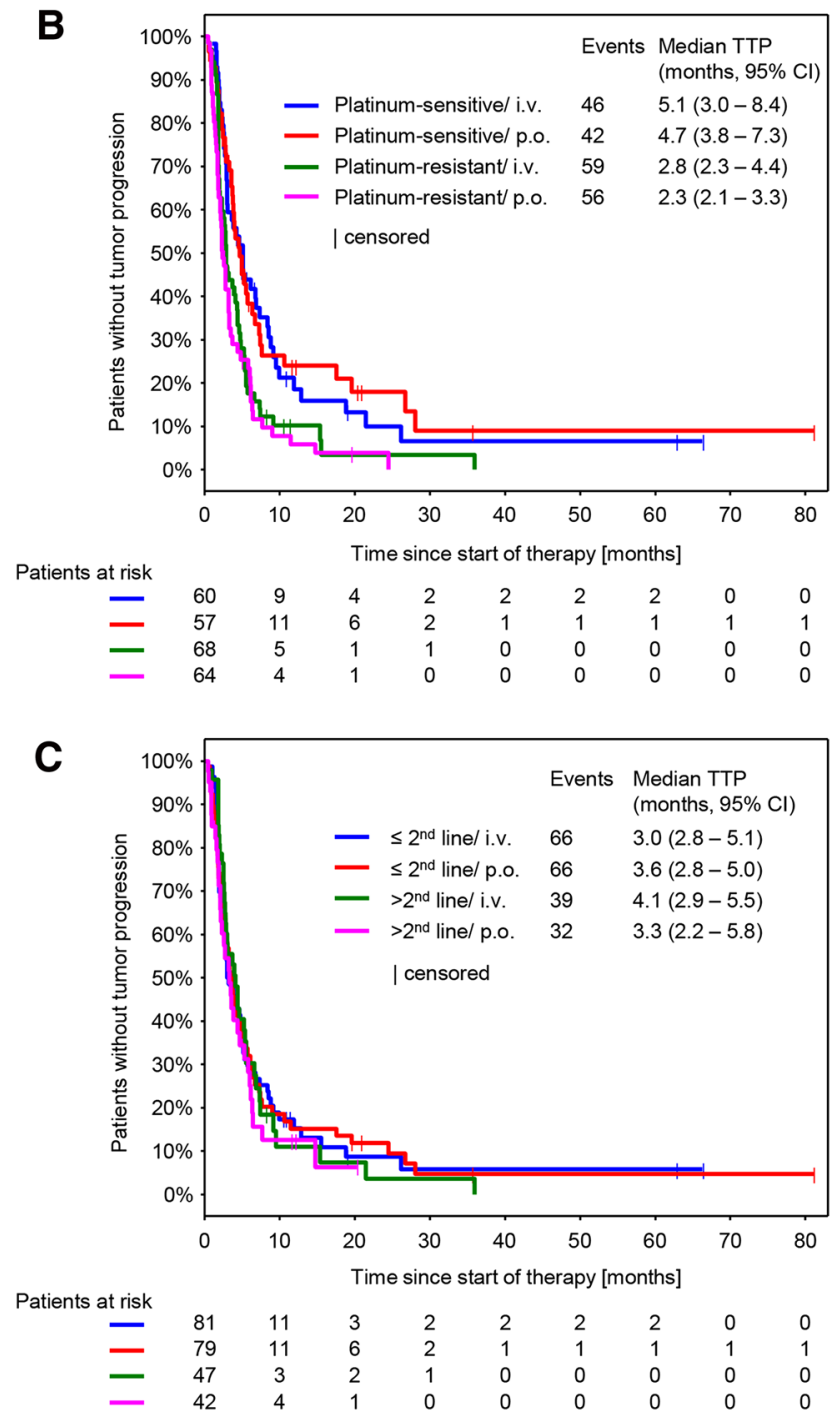

Fig. 2 Time to progression (TTP) (a) By treatment arm (b). By platinum sensitivity and treatment arm (c) By number of previous therapy lines and treatment arm. $H R$ Hazard ratio second-line treatment for platinum-resistant cancer in a patient collective with a median age of 61 years. Meden et al. (1997) investigated toxicities of oral treosulfan in patients receiving treosulfan as maintenance therapy after first-line chemotherapy. As in our study, in total leucopenia and thrombocytopenia were among the most frequently reported grade III/IV toxicities. The frequency of observed thrombocytopenia (7.2\%) is comparable with those published by Meier et al. (2009) (7.5\%) and Mahner et al. (2012) (4.0\%). In contrast, the frequency of leucopenia observed in the treosulfan i.v. arm in our study (3.9\%) is much lower than that reported by Meier et al. (2009) (18.3\%) and Mahner et al. (2012) (12.0\%). Additionally neutropenia was far less frequently observed in our study $(0.8 \%)$ than by Meier et al. (2009) (14.4\%) and Mahner et al. (2012) (8.0\%). Possible explanations for this difference in toxicity rates could be that Meier et al. used a more dose-dense treatment regimen $\left(7000 \mathrm{mg} / \mathrm{m}^{2} \mathrm{~d} 1 \mathrm{q} 3 \mathrm{w}\right)$, but patients in these study were less heavily pretreated.

No significant differences in disease control rate $(41.4 \%$ in the i.v. arm and $36.9 \%$ in the p.o. arm), time to progression (3.7 months in the i.v. arm and 3.5 months in the p.o. arm) or overall survival (13.6 months in the i.v. arm and 10.4 months in the p.o. arm) were observed in our study. In comparison to data from a first-line study by Reed et al. (2006) and a second-line study by Gropp et al. (1998) on intravenous treosulfan, disease control (Reed: 50.0\%; Gropp: 53.0\%) and time to progression (Reed: 5.0 months) are slightly lower in our study. However, in our study we were dealing with patients with many concomitant diseases in more advanced treatment lines that were older than patients investigated in the second-line study by Gropp et al. In our study, a nonsignificant trend toward a prolonged survival in the i.v. arm could be observed (13.6 vs. 10.4 months). But this comparison is due the very limited methodological limitations only hypothesis generating.

Interestingly the number of previous treatment lines (two or less vs. more than two) did not have an impact on any of the efficacy parameters. This indicates that also heavily pretreated patients benefit from treosulfan treatment to the same extent. A recent large study of the German AGO stated that patients with relapsed ovarian cancer seem to benefit form subsequent relapse treatment at the second to fourth recurrence (Hanker et al. 2012).

Due to several amendments throughout the entire term of the study, different definitions of how to count previous treatment lines were used. As we merged induction and reinduction therapy for analyses, there might be an underestimation of previous therapy lines. This has to be considered when comparing our results with other studies.

When comparing survival data of platinum-resistant patients in our study to data from a large phase III study (Pujade-Lauriane et al. 2014) in which ovarian cancer 


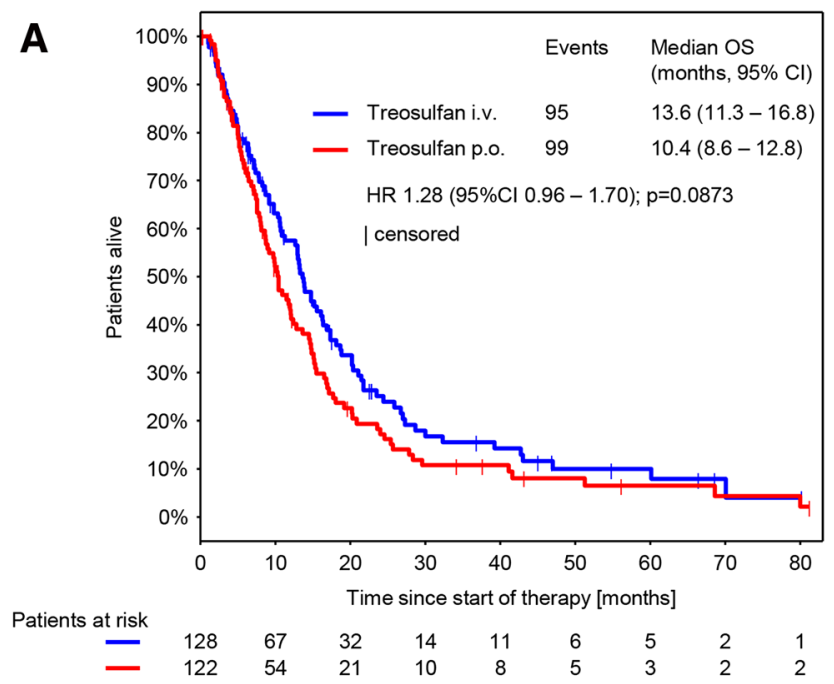

\section{B}

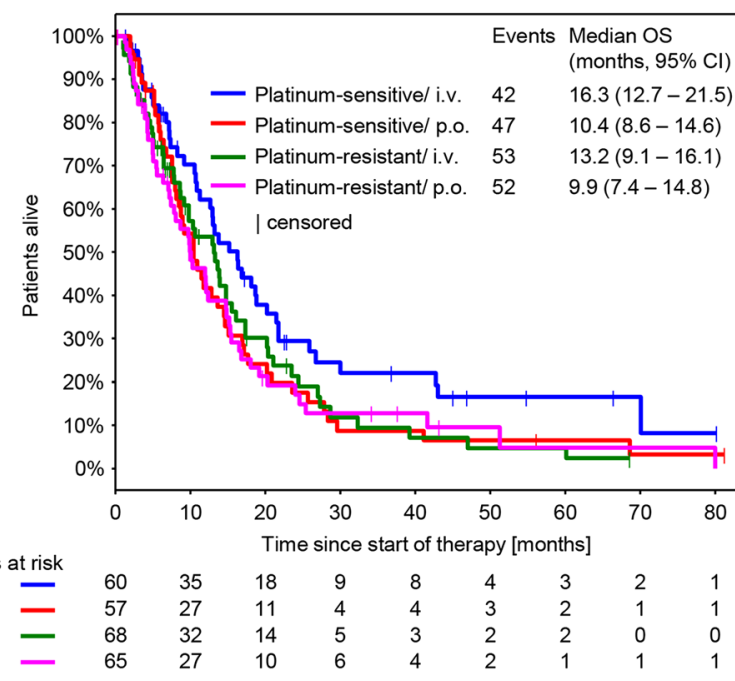

\section{C}

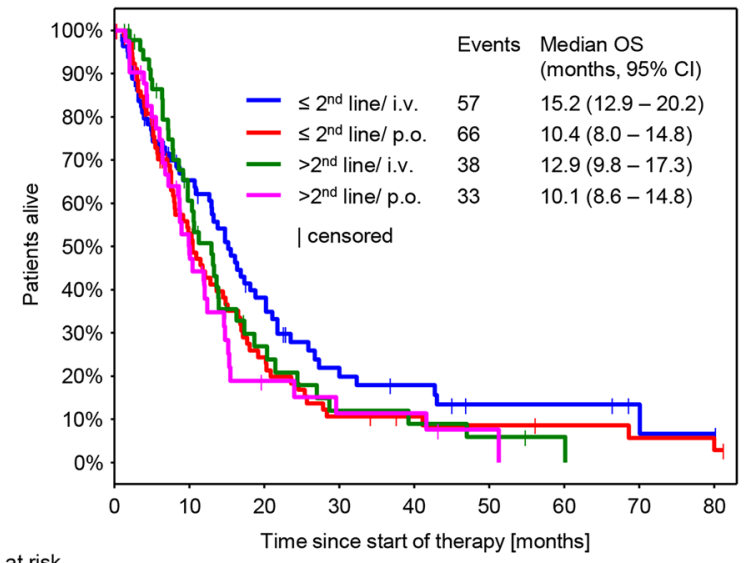

\begin{tabular}{cccccccccc} 
Patients at risk & \multicolumn{8}{c}{ Time since start of therapy [months] } \\
- & 81 & 42 & 23 & 10 & 8 & 4 & 4 & 2 & 1 \\
- & 80 & 37 & 16 & 7 & 5 & 4 & 3 & 2 & 2 \\
- & 47 & 25 & 9 & 4 & 3 & 2 & 1 & 0 & 0 \\
- & 42 & 17 & 5 & 3 & 3 & 1 & 0 & 0 & 0
\end{tabular}

Fig. 3 Overall survival (OS) (a) By treatment arm (b). By platinum sensitivity and treatment arm (c) By number of previous therapy lines and treatment arm. HR Hazard ratio; TTP Time to progression patients are treated with chemotherapy \pm bevacizumab in second and third line, overall survival of platinum-resistant patients treated with treosulfan in our study is within the same range as survival of platinum-resistant patients treated with chemotherapy only (11.9 vs. 13.3 months). Given the mild toxicity profile of treosulfan therapy and similar effect on disease survival, the treosulfan therapy seems to be an attractive option for treatment of the platinum-resistant recurrent disease.

The quality of life analysis did not show any significant differences in global health score or in selected subscales between the two treatment arms. In both arms a nonsignificant slight improvement in quality of life during therapy could be observed. However, due to many patients experiencing early progressive disease during therapy, the available data set shows a high dropout rate, limiting the meaningfulness of the analysis.

In summary, observed toxicities with oral and intravenous treosulfan were in the same range as in previous studies. Interestingly a higher number of leucopenia grade III/ IV occurred in the p.o. treatment arm. Apart from this, no additional significant differences in toxicity were observed between the treatment arms. CBR, TTP and OS data were comparable to previously reported data and did not differ significantly between both treatment arms. Heavy pretreatment did not detract from the benefit of treosulfan treatment. Patients with ROC who have frequently more gastrointestinal symptoms and polypharmacy due to co-morbidities may tolerate an i.v. treatment better. In a synopsis of efficacy and safety outcomes, there might be an advantage for i.v. application form for treosulfan treatment. Still, treatment with oral treosulfan remains a considerable option.

Acknowledgements We thank Melanie Frank for the conduction of the statistical analysis and Dr Judith Müller for providing medical writing services on behalf of iOMEDICO AG.

Funding The trial was supported by the North-Eastern German Society of Gynecological Oncology (NOGGO) and by medac GmbH.

\section{Compliance with ethical standards}

Conflict of interest Jalid Sehouli declares that he received an educational grant from medac. Michael Patrick Lux declares that he received honoraria for lectures, honoraria for editorial board membership of a journal as well as non-financial support for congress visit from medac. Tanja Trarbach declares that iOMEDICO received financial support from medac. Oliver Tomè, Oumar Camara, Ingo Bernhard Runnebaum, Hans Werner Tessen, Beate Rautenberg, Radoslav Chekerov and Gerald Gitsch declare that there are no conflicts of interest.

Ethical approval All procedures performed in studies involving human participants were in accordance with the ethical standards of the institutional and/or national research committee and with the 1964 Helsinki Declaration and its later amendments or comparable ethical standards. 
Informed consent Informed consent was obtained from all individual participants included in the study.

Open Access This article is distributed under the terms of the Creative Commons Attribution 4.0 International License (http://creativecommons.org/licenses/by/4.0/), which permits unrestricted use, distribution, and reproduction in any medium, provided you give appropriate credit to the original author(s) and the source, provide a link to the Creative Commons license, and indicate if changes were made.

\section{References}

Aghajanian C, Blank SV, Goff BA, Judson PL, Teneriello MG, Husain A et al (2012) OCEANS: a randomized, double-blind, placebo-controlled phase III trial of chemotherapy with or without bevacizumab in patients with platinum-sensitive recurrent epithelial ovarian, primary peritoneal, or fallopian tube cancer. $\mathbf{J}$ Clin Oncol 30:2039-2045. doi:10.1200/JCO.2012.42.0505

Boztug H, Zecca M, Sykora K-W, Veys P, Lankester A, Slatter M et al (2014) Treosulfan-based conditioning regimens for allogeneic HSCT in children with acute lymphoblastic leukaemia. Ann Hematol. doi:10.1007/s00277-014-2196-8

de Angelis R, Sant M, Coleman MP, Francisci S, Baili P, Pierannunzio D, Trama A, Visser O, Brenner H, Ardanaz E, Bielska-Lasota M, Engholm G, Nennecke A, Siesling S, Berrino F, Capocaccia R, EUROCARE-5 Working Group (2014) Cancer survival in Europe 1999-2007 by country and age: results of EUROCARE-5-a population-based study. Lancet Oncol 15(1):23-34. doi:10.1016/S1470-2045(13)70546-1

Ferlay J, Steliarova-Foucher E, Lortet-Tieulent J, Rosso S, Coebergh JWW, Comber H et al (2013) Cancer incidence and mortality patterns in Europe: estimates for 40 countries in 2012. Eur J Cancer 49:1374-1403. doi:10.1016/j.ejca.2012.12.027

Gropp M, Meier W, Hepp H (1998) Treosulfan as an effective second-line therapy in ovarian cancer. Gynecol Oncol 71:94-98. doi:10.1006/gyno.1998.5103

Hanker LC, Loibl S, Burchardi N, Pfisterer J, Meier W, Pujade-Lauraine $E$ et al (2012) The impact of second to sixth line therapy on survival of relapsed ovarian cancer after primary taxane/platinum-based therapy. Ann Oncol 23(10):2605-2612. doi:10.1093/ annonc/mds 203

Hilger RA, Jacek G, Oberhoff C, Kredtke S, Baumgart J, Seeber S et al (2000) Investigation of bioavailability and pharmacokinetics of treosulfan capsules in patients with relapsed ovarian cancer. Cancer Chemother Pharmacol 45:483-488. doi:10.1007/ s002800051023

Keldsen N, Madsen EL, Havsteen H, Kamby C, Laursen L, Sandberg E (1998) Oral treosulfan as second-line treatment in platinumresistant ovarian cancer: a phase II study. The Danish ovarian cancer study group. Gynecol Oncol 69:100-102

Köpf-Maier P, Sass G (1996) Antitumor activity of treosulfan in human lung carcinomas. Cancer Chemother Pharmacol $37: 211-221$
Liu G, Franssen E, Fitch MI, Warner E (1997) Patient preferences for oral versus intravenous palliative chemotherapy. JCO 15:110-115

Mahner S, Oskay-Özcelik G, Heidrich-Lorsbach E, Fuxius S, Sommer H, Klare P et al (2012) A prospective multicenter study of treosulfan in elderly patients with recurrent ovarian cancer: results of a planned safety analysis. J Cancer Res Clin Oncol 138:1413-1419. doi:10.1007/s00432-012-1221-3

McGuire WP, Hoskins WJ, Brady MF, Kucera PR, Partridge EE, Look KY et al (1996) Cyclophosphamide and cisplatin compared with paclitaxel and cisplatin in patients with stage III and stage IV ovarian cancer. N Engl J Med 334:1-6. doi:10.1056/ NEJM199601043340101

Meden H, Wittkop Y, Kuhn W (1997) Maintenance chemotherapy with oral treosulfan following first-line treatment in patients with advanced ovarian cancer: feasibility and toxicity. Anticancer Res 17:2221-2223

Meier W, du Bois A, Reuss A, Kuhn W, Olbricht S, Gropp M et al (2009) Topotecan versus treosulfan, an alkylating agent, in patients with epithelial ovarian cancer and relapse within 12 months following 1st-line platinum/paclitaxel chemotherapy. A prospectively randomized phase III trial by the Arbeitsgemeinschaft Gynaekologische Onkologie Ovarian Cancer Study Group (AGO-OVAR). Gynecol Oncol 114:199-205. doi:10.1016/j. ygyno.2009.04.026

Monk BJ, Herzog TJ, Kaye SB, Krasner CN, Vermorken JB, Muggia FM et al (2010) Trabectedin plus pegylated liposomal doxorubicin in recurrent ovarian cancer. J Clin Oncol 28:3107-3114. doi:10.1200/JCO.2009.25.4037

Oza AM, Cibula D, Benzaquen AO, Poole C, Mathijssen RHJ, Sonke GS et al (2015) Olaparib combined with chemotherapy for recurrent platinum-sensitive ovarian cancer: a randomised phase 2 trial. Lancet Oncol 16:87-97. doi:10.1016/ S1470-2045(14)71135-0

Pujade-Lauraine E, Wagner U, Aavall-Lundqvist E, Gebski V, Heywood M, Vasey PA et al (2010) Pegylated liposomal doxorubicin and carboplatin compared with paclitaxel and carboplatin for patients with platinum-sensitive ovarian cancer in late relapse. $\mathrm{J}$ Clin Oncol 28(20):3323-3329. doi:10.1200/JCO.2009.25.7519

Pujade-Lauraine E, Hilpert F, Weber B, Reuss A, Poveda A, Kristensen $G$ et al (2014) Bevacizumab combined with chemotherapy for platinum-resistant recurrent ovarian cancer: the AURELIA open-label randomized phase III trial. J Clin Oncol 32(13):1302-1308. doi:10.1200/JCO.2013.51.4489

Reed NS, Poole CJ, Coleman R, Parkin D, Graham JD, Kaye SB et al (2006) A randomised comparison of treosulfan and carboplatin in patients with ovarian cancer: a study by the Scottish Gynaecological Cancer Trials Group (SGCTG). Eur J Cancer 42(2):179185. doi:10.1016/j.ejca.2005.09.022

Sehouli J, Stengel D, Oskay-Oezcelik G, Zeimet AG, Sommer H, Klare P et al (2008) Nonplatinum topotecan combinations versus topotecan alone for recurrent ovarian cancer: results of a phase III study of the North-Eastern German Society of Gynecological Oncology Ovarian Cancer Study Group. J Clin Oncol 26(19):3176-3182. doi:10.1200/JCO.2007.15.1258 